\title{
Safety of Subclavian Venous Catheterization Using Anatomical Landmark Technique Versus Ultrasound-Guided Internal Jugular Central Venous Method Inserted in The Neuro-Intensive Care Unit
}

\section{Michael Eibach}

Goethe University Frankfurt

Fatma Kilinc

Goethe University Frankfurt

Florian Gessler

Goethe University Frankfurt

Ulrich Strouhal

Goethe University Frankfurt

\section{Volker Seifert}

Goethe University Frankfurt

Bedjan Behmanesh ( $\sim$ bedjan.behmanesh@gmail.com )

Goethe University Frankfurt

\section{Research Article}

Keywords: Central venous catheterization, Jugular vein, Subclavian vein, Ultrasound, Complication

Posted Date: February 13th, 2021

DOl: https://doi.org/10.21203/rs.3.rs-186209/v1

License: (c) (i) This work is licensed under a Creative Commons Attribution 4.0 International License.

Read Full License 


\section{Abstract}

\section{Background:}

Central venous (CV) catheterization is one of the important interventions used for various purposes. Accordingly, this study aimed to compare the complication rates of internal jugular vein (IJV) assisted by ultrasound with those of subclavian vein (SCV) catheterization using the anatomical landmark technique.

\section{Methods:}

Prospective, nonrandomized, single center study. Data from patients undergoing central venous catheterization either via IJV or SCV were recorded. The overall complication rate as well as number of attempts and catheter misplacement were analyzed comparing both methods

\section{Results:}

Data from 398 (IJV: 206; SCV:192) patients were analyzed. The rate of pneumothorax did not demonstrate a significant difference between the IJV (0.5\%) and SCV (2.1\%) groups, $p=0.2$. The success rate on the first attempt was similar in both groups $(64 \% ; p=1.0)$. More catheter misplacements were observed in the SCV group (4.7\%) than in the IJV group (3\%), $p=0.4$.

\section{Conclusions:}

Our results demonstrated that the complication rates of anatomical landmark guided SCV is very low and comparable to US guided IJV method.

\section{Introduction}

Central venous (CV) catheterization is an important procedure used for a number of purposes, such as fluid therapy, hemodynamic monitoring, administration of medication and nutrition. Although placement of a CV catheter is a routine procedure in intensive care medicine, emergency units and anesthesiology, acute severe complications, such as arterial puncture, hemothorax, pneumothorax or malposition may occur ${ }^{1,2}$.

Traditionally, CVC placement is performed using landmark techniques based on the knowledge of anatomic structures and palpation of arteries next to the veins. The use of ultrasound (US) has been proposed to reduce the number of CVC associated complications and to increase the safety and quality of CVC placement. Many studies have been published comparing the safety and efficacy of US-guided cannulation and landmark techniques of the same vein, either IJV or SCV ${ }^{2-6}$. To the best of our knowledge no previously data have been published evaluating the US-guided cannulation of the IJV compared to the catheterization of the SCV using landmark technique. Within this study we analyzed the safety, efficacy and potential benefit of a technique. 


\section{Material And Methods}

Within this prospective, nonrandomized study data from patients $>18$ years, who were scheduled to undergo major cranial (tumor surgery, aneurysmal clipping, trauma) or spine surgery that required CVC were assigned to the IJV group. In this group CVC were performed via the right or left IJV using ultrasound by neuroanesthesiologists/neurosurgeons with experience of performing the procedure. In these cases, the US-transducer was placed over the neck and venous flow was confirmed using color Doppler view. Once the vein was visible the needle was introduced in a plane perpendicular to the long axis of the transducer. After confirming blood aspiration and needle tip position inside the vein, the syringe was removed before a guide wire was introduced through the needle. After identifying the guidewire inside the jugular vein in a short- and long-axis ultrasound examination, the Seldinger technique was continued until catheter insertion and its position was confirmed by US.

Patients were admitted to our Neuro Intensive Care unit for neurosurgical treatment and need for central vein access were cannulated via the SCV without US using anatomical landmarks by neurosurgeons/neuroanesthesiologists. The infraclavicular approach was used for the SCV

Catheterization. After venous puncture was confirmed, the guidewire was advanced though the needle and into the vein. The needle was then removed while holding the guidewire in place. After placement of the guidewire the catheter was inserted and the position was confirmed by aspiration of venous blood. To proof the correct position of the catheter and exclude complications, such as pneumothorax or hemothorax chest X-rays were acquired after the procedure. The target position of the catheter tip was the superior vena cava.

Patients with impaired hemostatis, missing data and anatomical abnormalities were excluded.

\section{Outcome variables}

The primary outcome measure included arterial puncture (defined as any pulsatile blood reflux through the needle observed during the procedure); pneumothorax; and hemothorax. As secondary outcomes, we assessed the number of attempts to successfully achieve catheter placement, the number of the first attempt. The incidence of catheter misplacement, which was defined as the position of the catheter tip identified at any place other than the superior vena cava on the chest X-ray were recorded.

\section{Statistical Analysis}

All variables were tested for normality using the Shapiro-Wilk test. Considering the appropriateness of each test, either the Student's t test or Mann-Whitney U test was performed to compare continuous variables between the two groups. Categorical data were analyzed using the $\chi 2$ test or Fisher's exact test, as appropriate. A p value (two-sided in all tests) of $<0.05$ was considered significant. SPSS software, version 11.0, was used (SPSS Inc., Chicago, IL, USA).

\section{Study Approval}


This study was approved by the local ethics committee of the Goethe-University of Frankfurt. All methods were carried out in accordance with relevant guidelines and regulations. Informed consent was obtained from all participants.

\section{Results}

The overall number of patients being included and evaluated was 398. An internal jugular venous catheter (IJV) was placed in 206 (52\%) and a subclavian venous catheter in 192 (48\%) patients. Median age and sex the male/female distribution were significantly different. The basic characteristics of patents are summarized in Table 1.

Table 1

Basic patients' characteristics

\begin{tabular}{|llll|}
\hline & IJV & SCV & p-value \\
& $(\mathbf{N}=\mathbf{2 0 6})$ & $\mathbf{( N = 1 9 2 )}$ & \\
\hline Age (median, $\mathrm{y})$ & 60 & 64 & 0.03 \\
\hline Male & $110(53.4 \%)$ & $123(62.1 \%)$ & 0.03 \\
\hline Female & $96(46.6 \%)$ & $69(35.9 \%)$ & \\
\hline High (median, cm) & 170 & 173 & 0.7 \\
\hline Weight (median, $\mathrm{Kg})$ & 75 & 80 & 0.2 \\
\hline BMI (median, $\left.\mathrm{cm} / \mathrm{kg}^{2}\right)$ & 25.1 & 25.7 & 0.9 \\
\hline Cranial surgery & $179(86.9 \%)$ & $170(88.5 \%)$ & 0.6 \\
\hline Spine surgery & $27(13.1 \%)$ & $22(11.5 \%)$ & \\
\hline
\end{tabular}

The overall incidence of complications was very low in both approaches and did not differ significantly between SCV and IJV cannulations ( $2.9 \%$ vs. $7.3 \%, p=0.06)$. Whereas severe complications, such as pneumothorax occurred with $0.5 \%$ in the IJV group and $2.1 \%$ in the SCV group, $p=0.2$, arterial puncture as a minor complication has been observed more often in the SCV group ( $2.4 \%$ vs. $5.2 \%, p=0.2)$, as well. The number of catheter misplacement was higher in the SCV group than in the IJV group ( $3 \% \mathrm{vs.} 4.7 \%, p$ $=0.4)$, Fig. 1 .

The overall success rate was $99 \%$ in IJV group and $96.9 \%$ in the SCV group, $p=0.2$, of which $64 \%$ were placed on first attempt in both groups. The average number of attempts for catheter insertion was almost identical in the SCV group compared to IJV group, $p=0.5$. Most IJV central venous catheters were inserted in the right vein compared to the SCV catheter, $(87 \%$ vs. $70.3 \% ; p=0.001)$, Table 2. 
Table 2

Complications and details of catheter placement

\begin{tabular}{|llll|}
\hline & IJV & SCV & p-value \\
& $(\mathbf{N}=\mathbf{2 0 6})$ & $\mathbf{( N = 1 9 2 )}$ & \\
\hline Median number of attempts & 1 & 1 & 0.5 \\
\hline Success, \% & 99 & 96.9 & 0.2 \\
\hline First attempt & $132(64 \%)$ & $122(64 \%)$ & 1.0 \\
\hline second attempt & $69(33.5 \%)$ & $50(26 \%)$ & 0.1 \\
\hline Third attempt & $3(1.5 \%)$ & $9(4.7 \%)$ & 0.2 \\
\hline$>$ 3rd attempt & $2(1 \%)$ & $11(5.7 \%)$ & 0.004 \\
\hline Right side & $179(87 \%)$ & $135(70.3 \%)$ & 0.0001 \\
\hline Left side & $27(13 \%)$ & $57(29.7 \%)$ & \\
\hline Malposition & $6(3 \%)$ & $9(4.7 \%)$ & 0.4 \\
\hline Unsuccessful attempt & $2(1 \%)$ & $6(3 \%)$ & 0.2 \\
\hline Complications & & & \\
\hline Arterial puncture & $5(2.4 \%)$ & $10(5.2 \%)$ & 0.2 \\
\hline Pneumothorax & $1(0.5 \%)$ & $4(2.1 \%)$ & 0.2 \\
\hline Hemothorax & 0 & 0 & \\
\hline
\end{tabular}

\section{Discussion}

Several advantages of the subclavian vein for central access include its large diameter, absence of valves, and ability to remain patent. Subclavian catheterization also carries a lower risk of catheterrelated infection and thrombosis than femoral or internal jugular vein catheterization ${ }^{1,7,8}$. Furthermore, this approach offers a reduced rate of infection, thrombosis, improved patient comfort and tolerance and the cannulation is often easier in hypovolaemic patients ${ }^{9-11}$. Moreover, to guarantee access to the neck and ensure venous blood drainage from the brain the SCV approach provides an important advantage in the setting of neurosurgical intensive care units.

Since CV catherization remains an important procedure for patients being treated within intensive care units, the safety of the procedure, reduction of the complications rate is still an important issue. Many studies have been published comparing US-guided techniques and landmark-based techniques puncturing either IJV or SCV and supporting the superiority of US-guided techniques in reduction of complications and attempts until successful catheterization. Nevertheless, to date, no data exists reporting safety and complication rates puncturing IJV with US-support and SCV using landmark 
technique. With regard to complication rate, no significant differences were demonstrated between the SCV and IJV groups. The only significant parameter was the number of attempts more than 3 , which was higher in the SCV group. These findings are in line with previous published data, which underline the almost similar complications rates of mechanical complications, but the superiority of the US -guided technique in terms of average access time and number of attempts between internal jugular and subclavian venous catheterization ${ }^{12-14}$.

The use of CVCs may be associated with adverse effects to patients ${ }^{1}$. Mechanical complications are reported to occur up to $20 \%$ of patients, infectious and thrombotic complications up to $30 \%{ }^{15,16}$. Thus, the procedure of $\mathrm{CVC}$ remains a high complicative approach, which should be indicated with great attention and be performed under safe conditions.

So, the research in terms of safety approach and the use of technical support are still important and of need. So, we tried to provide further insight in this field by presenting our achieved data. One aspect should be noticed, that the cannulation of the SCV using US require greater technical US skill due to its route beneath the clavicle ${ }^{6,17,18}$. Nevertheless, we could show, that the SCV access remains an alternative approach in neurosurgically treated patients.

\section{Conclusions}

Within this study we demonstrated that the subclavian vein access using the landmark technique is safe compared to ultrasound-guided internal jugular vein catheterization. Nevertheless, the number of attempts and access time was in favor of the IJV method.

\section{Declarations}

\section{Data Availability.}

The datasets generated during and/or analyzed during the current study are available from the corresponding author on reasonable request.

\section{Author Contributions:}

M.E.: study enrollment, data collection, writing and formatting manuscript, submission

F.K.: data collection, interpretation of the data

F.G.: data collection, follow-up examinations

U.S.:. interpretation of the data, data collection

V.S.: Supervising the study, supervising the study 
B.B.: Supervising the study, senior surgeon

\section{Competing financial interests}

The authors declare no competing financial interests.

\section{References}

1. McGee, D. C. \& Gould, M. K. Preventing complications of central venous catheterization. The New England journal of medicine.348, 1123-1133 (2003).

2. Ruesch, S., Walder, B. \& Tramèr, M. R. Complications of central venous catheters: internal jugular versus subclavian access-a systematic review. Critical care medicine.30, 454-460 (2002).

3. Brass, P., Hellmich, M., Kolodziej, L., Schick, G. \& Smith, A. F. Ultrasound guidance versus anatomical landmarks for subclavian or femoral vein catheterization. The Cochrane database of systematic reviews.1, CD011447 (2015).

4. Kim, Y. O. et al. Safety and feasibility of ultrasound-guided placement of peripherally inserted central catheter performed by neurointensivist in neurosurgery intensive care unit. PloS one.14, e0217641 (2019).

5. Gordon, A. C., Saliken, J. C., Johns, D., Owen, R. \& Gray, R. R. US-guided puncture of the internal jugular vein: complications and anatomic considerations. Journal of vascular and interventional radiology: JVIR9,333-8

6. Saugel, B., Scheeren, T. W. L. \& Teboul, J. L. Ultrasound-guided central venous catheter placement: a structured review and recommendations for clinical practice. Critical care (London, England).21, 225 (2017).

7. Defalque, R. J. Subclavian venipuncture: a review.Anesthesia and analgesia47,677-82

8. Yoffa, D. Supraclavicular subclavian venepuncture and catheterisation. Lancet (London, England).2, 614-617 (1965).

9. Parienti, J. J. et al. Intravascular Complications of Central Venous Catheterization by Insertion Site. The New England journal of medicine.373, 1220-1229 (2015).

10. Frykholm, P. et al. Clinical guidelines on central venous catheterisation. Swedish Society of Anaesthesiology and Intensive Care Medicine. Acta anaesthesiologica Scandinavica.58, 508-524 (2014).

11. Parienti, J. J. et al. Meta-analysis of subclavian insertion and nontunneled central venous catheterassociated infection risk reduction in critically ill adults. Critical care medicine.40, 1627-1634 (2012).

12. Fragou, M. et al. Real-time ultrasound-guided subclavian vein cannulation versus the landmark method in critical care patients: a prospective randomized study. Critical care medicine.39, 16071612 (2011). 
13. Sidoti, A. et al. Ultrasound- versus landmark-guided subclavian vein catheterization: a prospective observational study from a tertiary referral hospital. Scientific reports.9, 12248 (2019).

14. Gualtieri, E., Deppe, S. A., Sipperly, M. E. \& Thompson, D. R. Subclavian venous catheterization: greater success rate for less experienced operators using ultrasound guidance. Critical care medicine.23, 692-697 (1995).

15. Richards, M. J., Edwards, J. R., Culver, D. H. \& Gaynes, R. P. Nosocomial infections in medical intensive care units in the United States. National Nosocomial Infections Surveillance System. Critical care medicine.27, 887-892 (1999).

16. Merrer, J. et al. Complications of femoral and subclavian venous catheterization in critically ill patients: a randomized controlled trial. JAMA.286, 700-707 (2001).

17. Griswold-Theodorson, S., Farabaugh, E., Handly, N., McGrath, T. \& Wagner, D. Subclavian central venous catheters and ultrasound guidance: policy vs practice. The journal of vascular access $14,104-$ 10

18. Bowdle, A. Vascular complications of central venous catheter placement: evidence-based methods for prevention and treatment. Journal of cardiothoracic and vascular anesthesia.28, 358-368 (2014).

\section{Figures}
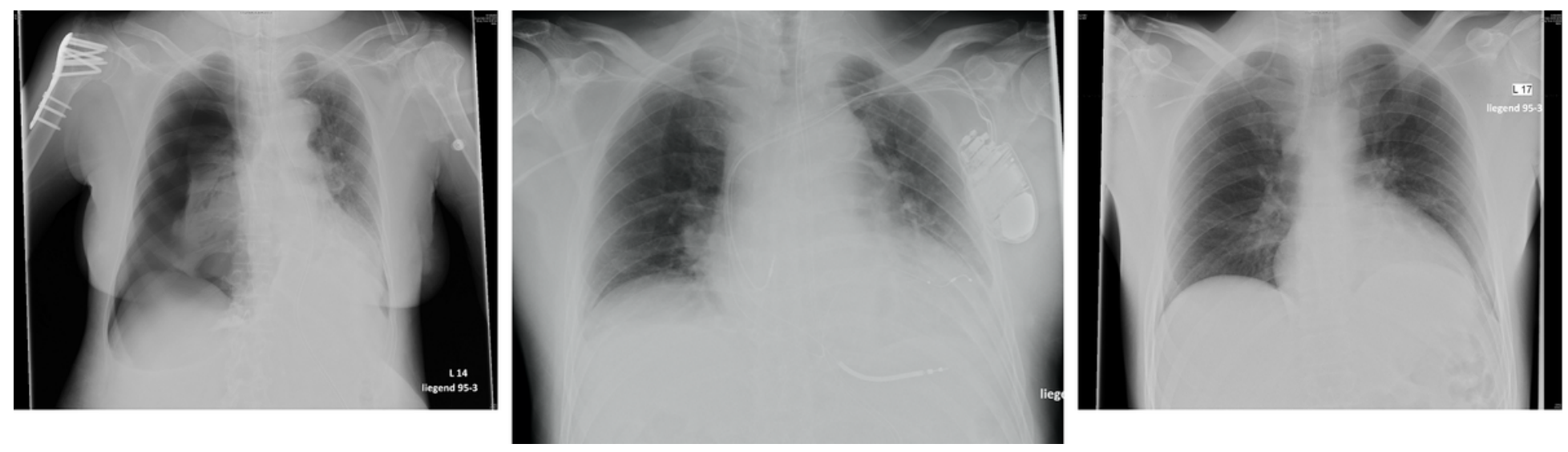

\section{Figure 1}

Procedure-related complications. Pneumothorax (a), catheter malposition to internal jugular vein (b) and right subclavian vein (c) 